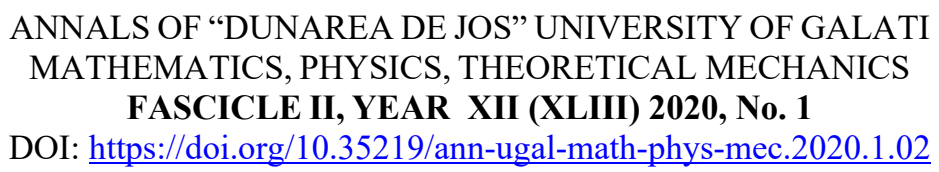

\title{
Differentiation of brain metastases in MRI image using the first and second-order statistical features
}

\author{
Lenuța Pană ${ }^{1}$, Simona Moldovanu ${ }^{2 *}$, Luminița Moraru ${ }^{1}$ \\ ${ }^{I}$ Faculty of Science and Environment, "Dunărea de Jos" University of Galați, 47 Domnească Street, RO- \\ 800008, Galați, Romania \\ 2 Faculty of Automation, Computers Sciences, Electrical Engineering and Electronics, "Dunărea de Jos" \\ University of Galați ,2 Stiinței Street, RO-800210, Galați, Romania \\ *Corresponding author: simona.moldoveanu@ugal.ro
}

\begin{abstract}
The brain is highly susceptible to metastases from lung cancer. The segmentation and detection of brain metastases are the main goals for the management of patients with brain metastases and a MRI technique that uses the image signal contrast between tissues rather than their absolute signal intensities is a recommended approach. This paper proposes a specific quantification method for the most relevant first and second-order features computed for brain images acquired as T2-weighted and PD (proton density). T2-w sequences are useful for detecting high-signal tumor infiltration whilst PD (proton density) sequences performed a fat suppression being an intermediate sequence between T1-w and T2-w and share common features of both. Based on the firstorder histogram (the gray-level distribution of the image) and on texture-related information provided by the cooccurrence matrix, features like skewness, kurtosis or entropy, and energy were computed in terms of their discriminatory power for a better clinical investigation of MRI images. Our analysis provides for a smaller number of relevant and distinguishable features and the computational task is at a reasonable level.
\end{abstract}

Keywords: Kurtosis, Skewness, Entropy, Energy.

\section{INTRODUCTION}

The segmentation and detection of brain metastases are the main goals for the management of patients with brain metastases. MRI is preferred over other imaging techniques as X-ray, CT, ultrasound for obtaining high contrast medical images. MRI parameters such as T1, T1 contrast, T2, FLAIR, PD (proton density) are commonly used for detecting and diagnosing several neurological disorders such as stroke, cysts, tumor, Parkinson's, and Alzheimer's diseases [1].

A tumor is a mass of tissue that grows out of control of the normal forces that regulate growth. Brain tumors are very heterogeneous in terms of shape, color, texture, and position and they often deform other nearby anatomical structures. The presence of a tumor in the human brain disturbs the brain sagittal symmetry. Brain tumors can be divided into primary or secondary based on the tumor's beginning or benign or malignant according to the malignancy. Primary brain tumors originate in the cells of the brain or in the surrounding tissues of the brain. Metastatic brain tumors emerge in different body parts (like breast or lungs) and migrate to the brain [2].

The MRI technique allows reaching a greater accuracy in characterizing various brain tissues (normal or abnormal). A large number of pattern recognition techniques have enabled experts to extract valuable information from the displayed texture and provide real support for an accurate diagnosis [3]. Aggarwal et al. [4] analyzed both normal brain and Alzheimer's disease MRI images in sequence T2-weighted. They investigated the following features: first and second-order statistics 
(FSStat), Daubechies-4 (Db4) and Haar in combination with PCA (HaarPCA) and classifiers such as SVM with linear kernel (SVM-L), polynomial kernel (SVM-P) and radial kernel (SVM-R), K-nearest neighbor $(\mathrm{KNN})$ and Levenberg-Marquardt Neural Classifier (LMNC). The best classification accuracy of $98.96 \%$ and sensitivity of $98.96 \%$ were reported for SVM-L. The rest of the classifiers show fair performance results. Kunimatsu et al. [5] investigated the differences between glioblastoma and primary central nervous system lymphoma using MR image-based texture features. Their analysis was based on the first-order features (histogram) and second-order features provided by the cooccurrence matrix, run-length matrix, gray-level size zone matrices, and multiple gray level size zone matrix. Their study has some limitations: a small number of patients, some texture features did not follow the normal distribution, did not make a comparison between results with texture analysis, and those with the human visual assessment. Priya et al. [6] used various SVM classifiers and different statistical feature sets such as first-order features, second-order features, and together to classify and grade four brain tumor types. Their results indicated that when the second-order features were used independently an accuracy of $85 \%$ and $78.26 \%$ was achieved, whereas for the first-order features used alone the accuracy was $65.517 \%$ and $62.31 \%$, respectively. When a combination of both features has been used the accuracy became $84.48 \%$ and $68.1 \%$, respectively. Zacharaki et al. [7] used a binary SVM to investigate and differentiate between several common brain tumors. Specifically, they used tumor shape, image intensity characteristics and Gabor texture features to differentiate between tumor types. They achieved an accuracy of $85 \%$ for distinguishing metastases from gliomas and $88 \%$ to distinguish the high grade from low-grade neoplasms.

In this study, the first-order (skewness and kurtosis) and second-order (entropy and energy) features are investigated by using PD-w (proton density) and T2-w (T2-weighted type) brain images of healthy patients and patients diagnosed with metastatic bronchogenic carcinoma (MBC). In the preprocessing step, a median filter is used for noise and distortion removal but with preservation of the edges. The methods used in this study are discussed in section 2 and results and discussion are presented in section 3 and the conclusions are presented in section 4 .

\section{METHODS}

\subsection{Mathematical approaches}

a) Median filter

For a grayscale image I with $x_{i, j}$ intensity values, and a window $W$ over which the filter is applied with the coordinates of the recently processed pixel $(i, j)$, the two-dimensional median filter is defined as [8]:

$$
\mathrm{y}_{\mathrm{i}, \mathrm{j}}=\underset{(r, s) \in W}{\operatorname{median}}\left(\mathrm{x}_{\mathrm{i}+\mathrm{r}, \mathrm{j}+\mathrm{s}}\right)
$$

\section{b) Skull stripping}

In order to eliminate all non-brain tissues from brain MRI, such as skull, fat, skin, etc., a skull stripping algorithm based on mathematical morphology is used $[9,10]$.

The skull-stripping operation is performed based on the following morphological operations:

I. Image binarization using a threshold value determined from the gray level histogram;

II. Removal of the small objects in the image;

III. Filling the gaps in the image and forming the binary mask;

IV. Erosion of the binary mask with a disk-type structuring element.

V. Overlaid the binarized mask over the gray level image.

An erosion operation of binary image A by the structuring element, is given by $[9,10]$ :

$$
A \Theta B=\{(i, j): B \subseteq A\}
$$


where $(i, j)$ denotes the center pixel of the structuring element and the structural element B is a disc with a radius of 5 .

c) Watershed segmentation

Watershed segmentation is a region-based segmentation technique. It considers the gradient map of the image as a topographic relief map and segments the image as a dam, i.e. the gradient magnitude corresponds to the altitude. The higher values of gradient magnitude are peaks and small values are assigned to the valleys. The segmented regions are called catchment basins.

d) Morphological reconstruction

To overcome the limits of erosion followed by dilatation morphological operations related to the structuring element similarity with the shapes of the objects, the opening by reconstruction algorithm is used. This method optimally restores the original shapes of the objects after the erosion operation is performed $[11,12]$.

The opening by reconstruction of a gray-scale image $\mathrm{A}(\mathrm{x}, \mathrm{y})$ using a gray-scale structuring element $\mathrm{B}(\mathrm{s}, \mathrm{t})$, is defined as,

$$
A \circ B \subseteq A \subseteq A \bullet B
$$

where $A \circ B$ is the morphological opening operation defined as

$$
A \circ B=(A \odot B) \oplus B
$$

and $A \bullet B$ is the morphological closing operation defined as

$$
A \cdot B=(A \oplus B) \odot B
$$

$\oplus$ denotes the dilation operation and $\Theta$ the morphological erosion operation.

The sharpness $\operatorname{Ed}(\mathrm{A})$ or dilation residue edge detector of the image $\mathrm{A}$ is defined as difference pixels set of the dilation domain of $\mathrm{A}$ and the domain of $\mathrm{A}$. $\mathrm{Ed}(\mathrm{A})$ is used to extract the contour feature details and it is defined as

$$
E_{d}(A)=(A \oplus B) \Theta A
$$

The structural element B is a disc with a radius of 5 .

\section{b) The first-order features}

The skewness and the kurtosis are intensity features and measure the asymmetry and the peakedness (or the heaviness of the tails) of the distribution, respectively $[13,14]$. The skewness is defined as,

$$
\gamma_{1}=E\left[\left(\frac{X-\mu}{\sigma}\right)^{3}\right]=\frac{\frac{1}{N^{2}} \sum_{x, y}(I(x, y)-\mu)^{3}}{\sigma^{3}}
$$

The kurtosis is defined as,

$$
\gamma_{2}=E\left[\left(\frac{X-\mu}{\sigma}\right)^{4}\right]-3=\frac{\frac{1}{N^{2}} \sum_{x, y}(I(x, y)-\mu)^{4}}{\sigma^{4}}-3
$$

where $\mu$ is mean the 1 -st moment and $\sigma$ is standard deviation. 
c) The second-order features are devoted to the joint probability occurrence of a specific pair of gray values for a pair of pixels randomly placed. Usually, the $2^{\text {nd }}$ order features are computable from the gray level co-occurrence matrices being texture features $C_{d}(i, j)$ constructed for an image $I$ with the pair of gray levels $\mathrm{i}, \mathrm{j}$ that are placed at a distance $\boldsymbol{d}$ [13],

$$
C_{d}(i, j)=\sum x, y \begin{cases}1, & I(x, y)=i, \quad I(x+d x, y+d y)=j \\ 0, & \text { otherwise }\end{cases}
$$

here, $\boldsymbol{d}=(d x, d y)$ denotes the displacement vector. Entropy $(E)$ and energy $(E N)$ are features related to orderliness (regularity or commonness) of the data and are defined as [13].

\begin{tabular}{|l|l|}
\hline$E=-\sum_{i, j} C(i, j) \ln C(i, j)$ & (10) \\
\hline$E N=\sum_{i, j} C(i, j)^{2}$ &
\end{tabular}

\subsection{SUBJECTS, IMAGE ACQUISITION AND POST-PROCESSING}

All data were analyzed using a computer with the following specifications: CPU Inter (R) Core (TM) i7-8550U @ 1.80 GHz CPU; memory (RAM) 8 GB DDR4; video card GeForce MX150 4 GB; hard disk 256 GB SSD, Windows 10, 64-byte. The programming environment is MATLAB R2017a, Image Processing toolbox (MathWorks Inc. Natick, MA). The image dataset was downloaded from the Harvard's Whole Brain Atlas website for free [15]. Experiments are conducted on a dataset that contains images of patients with MBC (metastatic bronchogenic carcinoma) and $\mathrm{H}$ (healthy) patients. The study includes 60 PD-w and T2-w images (256 x 256 pixels); 15 images PD-w and 15 images T2-w for patients diagnosed with MBC and 15 images PD-w and 15 images T2-w for $\mathrm{H}$ patients.

\subsection{FLOW CHART}

The proposed algorithm involves the following steps and T2-w image for a patient with MBC:

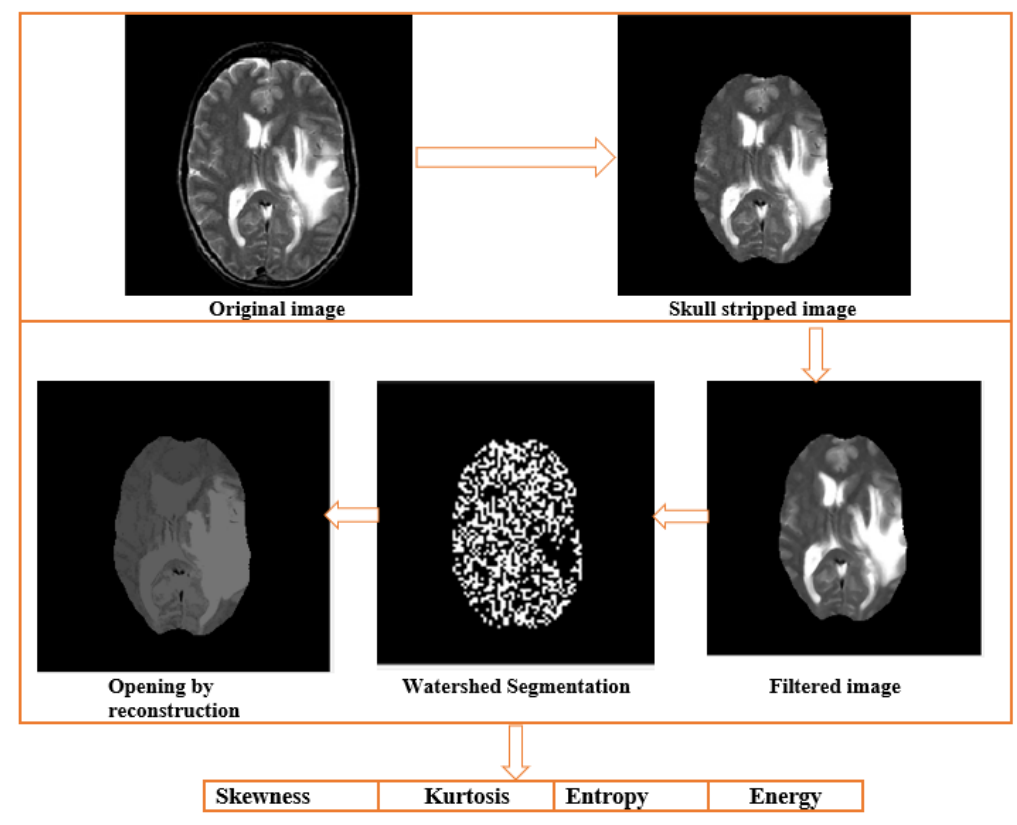

Figure 1. Flow chart of the proposed method 


\section{RESULTS AND DISCUSSION}

The problem we are dealing with is an assessment of the capability of the first and secondorder statistics features to distinguish healthy brain from brain metastases images, acquired as $\mathrm{T} 2-\mathrm{w}$ weighted and PD-w (proton density). In other words, we are interested in finding the potential for useful features.

Based on the histogram (the gray-level distribution in the image) and on texture-related information provided by the co-occurrence matrix, features like skewness, kurtosis, or entropy and energy were computed. There were denoted as PDH, the PD-w images and T2H, the T2-w images for healthy patients, respectively. Similarly, PDMBC is for PD-w images and T2MBC for T2-w images belonging to the MBC patients for healthy patients. The probability of occurrence for the first and second-order features using the histogram plot is displayed in Figure 2.

Also, Table 1 summarizes the results of mean and standard deviations of the first and secondorder features, for both patient groups.

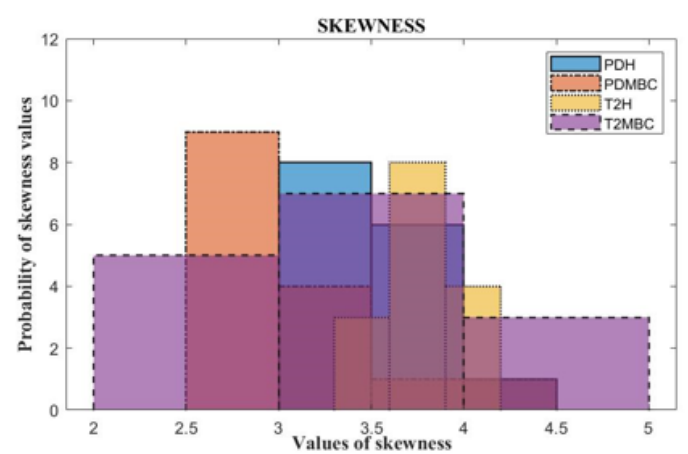

a)

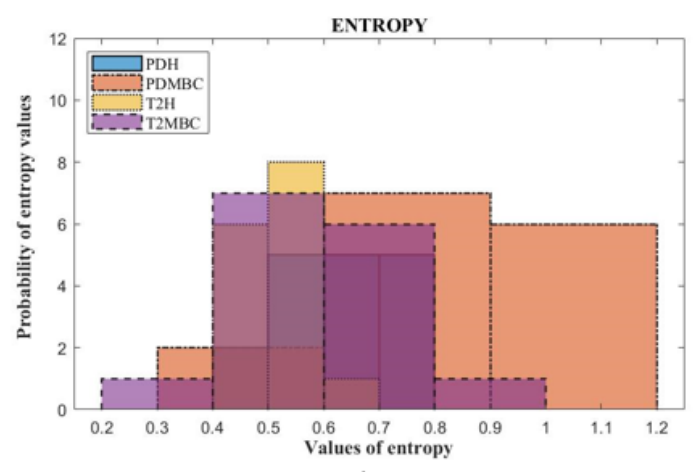

c)

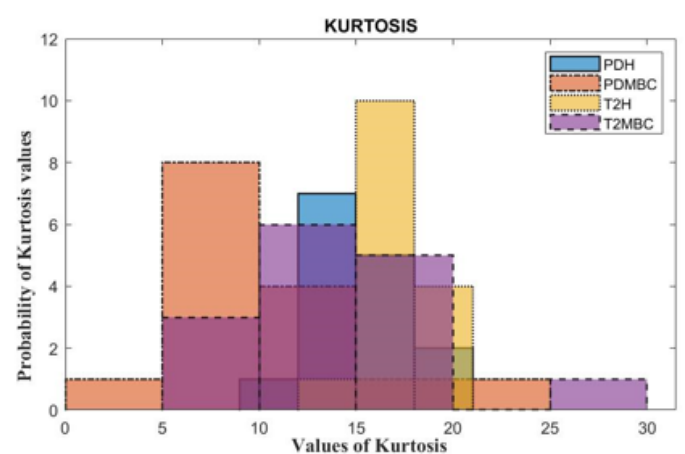

b)

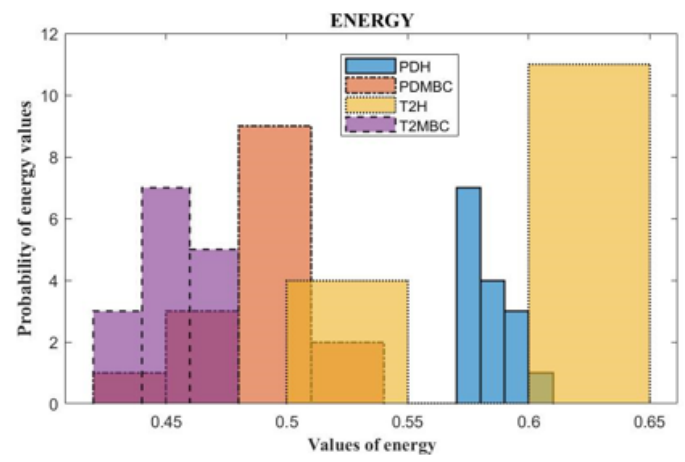

d)

Figure 2. Probability of occurrence for values (a) Skewness, (b) Kurtosis, (c) Entropy, (d) Energy vs. their determined values for healthy patients (T2H and PDH) and MBC patients (T2MBC and $P D M B C)$, respectively

In fig. 2a, the results indicate the skewness values for PDH images in a narrow range of values $[3 ; 4]$ with a higher value of 8 in the range [3; 3.5]; for PDMBC images they are from 2.5 to 4.5 with a higher value of 9 in the range $[2.5 ; 3]$. The larger values of skewness were obtained for PDMBC images and this finding indicates that the highest level of asymmetry occurs for MBC images. Similarly, the skewness values for T2H images range from 3.7 to 4.2 with a larger value of 8 in the interval $[3.6,3.9]$. In the case of T2MBC images, it is easily observed that they cover the whole range.

The kurtosis values (fig. 2b) for PDH images are in a wide range of values [9;21] with a higher value 7; for PDMBC images they are in an even wider range of values $[0 ; 25]$ with the higher value of 8 in the range $[5 ; 10]$. This indicates that the kurtosis feature has a strong asymmetry to the 
right. The kurtosis values for $\mathrm{T} 2 \mathrm{H}$ images are displayed in an even narrower range of values $[12 ; 20]$ and for the T2MBC images, the range of values is $[9 ; 30]$.

The results in figure 2c indicate the entropy values for PDH images are in a narrow range of values $[0.4 ; 0.8]$ with a higher value 5 in three ranges $[0.5 ; 0.6],[0.6 ; 0.7]$ and $[0.7 ; 0.8]$; for PDMBC images in a wide range of values $[0.3 ; 1.2]$, with the higher value of 7 . Similarly, the entropy values for T2H images are in a narrow range $[0.4 ; 0.70]$ and display the higher value of 8 among the studied sample; for T2MBC images they are in an even wider range of values $[0.2 ; 1]$.

The energy values (fig. $2 \mathrm{~d}$ ) for PDH images display in a narrow range of values $[0.57 ; 0.61]$ and show the higher value 7; for PDMBC images the energy is in a wide range of values range [0.15; $0,54]$. In the case of T2H images. The energy values are from 0.5 to 0.65 , with a higher value of 11 among the studied sample; for the T2MBC images, they are in the range $[0.15 ; 0.48]$ with the higher value 7 in the range $[0.44 ; 0.47]$. The energy values computed for patients with MBC and for PD type are separated for the rest of the values. Higher bins values of kurtosis (fig. 2 b), entropy (fig. 2c) and energy (fig. $2 \mathrm{~d}$ ) indicated a good differentiation between $\mathrm{H}$ and $\mathrm{MBC}$ patients for $\mathrm{T} 2-\mathrm{w}$ images.

Tabel 1. Mean \pm standard deviation of the first and second-order statistics features vs. image types and patient classes

\begin{tabular}{|l|l|l|l|l|}
\hline & Skewness & Kurtosis & Entropy & Energy \\
\hline PDH images & $3.550 \pm 0.471$ & $14.874 \pm 2.149$ & $0.612 \pm 0.093$ & $0.588 \pm 0.009$ \\
\hline PDMBC images & $3.010 \pm 0.533$ & $10.736 \pm 4.312$ & $0.810 \pm 0.189$ & $0.481 \pm 0.020$ \\
\hline T2H images & $1.862 \pm 0.001$ & $16.711 \pm 1.775$ & $0.521 \pm 0.062$ & $0.580 \pm 0.050$ \\
\hline T2MBC images & $1.866 \pm 0.002$ & $15.132 \pm 5.113$ & $0.583 \pm 0.149$ & $0.448 \pm 0.015$ \\
\hline
\end{tabular}

The data in Table 1 indicates how accurate is the mean of the first and second-order statistics features of the MBC patients compared to the mean values of the healthy patients. The standard deviation increases for the kurtosis and entropy features, for MBC patients. This finding could indicate the pathological condition of the brain. Our results indicate the kurtosis and entropy ability to detect metastatic pixels.

\section{CONCLUSIONS}

Making a diagnosis is essential to clinical medicine and the MRI plays an important role in analyzing various brain diseases. Our results indicate the ability of kurtosis and entropy features to detect metastatic pixels, for MRI images of both PD and T2-w type. In conclusion, our study demonstrates the potential use of first and second-order features in metastatic pixels detection.

\section{References}

1. Chandrasekaran R., Hamizhvani T. R.T, Sowmya Y. K., Babu B., Joseph J. E., JosphineArockiaDhivya A., MRI Tumor Segmentation - An Application Approach, International Journal of Pure and Applied Mathematics, 119(18) (2018) 3149-3163.

2. Roy S.,. Bandyopadhyay S. K, Detection and Quantification of Brain Tumor from MRI of Brain and it's Symmetric Analysis, International Journal of Information and Communication Technology Research, 2(6) (2012) 477-483.

3. Pantelis G., Dionisis C., Ioannis K., Dimitris G., Emmanouil A., Spiros K., Koralia S., Menelaos M., George N., Ekaterini S., Enhancing the discrimination accuracy between metastases, gliomas and meningiomas on brain MRI by volumetric textural features and 
ensemble pattern recognition methods, Journal Magnetic Resonance Imaging, 27(1) (2009) $120-130$.

4. Aggarwal N. and Agrawal R. K., First and Second Order Statistics Features for Classification of Magnetic Resonance Brain Images, Journal of Signal and Information Processing, 3(2) (2012) 146-153.

5. Kunimatsu A., Kunimatsu N., Kamiya K., Watadani T., Mori H., Abe O., Comparison between Glioblastoma and Primary Central Nervous System Lymphoma Using MR Imagebased Texture Analysis, Journal of Magnetic Resonance in Medical Sciences, 17(1) (2018) $50-57$.

6. K. M. Priya, S. Kavitha, B. Bharathi, Brain tumor types and grades classification based on statistical feature set using support vector machine, 10th International Conference on Intelligent Systems and Control (ISCO), Coimbatore, India, pp. 60-64, 2016.

7. Zacharaki E.I., Wang S., Chawla S., Yoo D.S., Wolf R., Methem R.E., Davatzikos C., Classification of brain tumor type and grade using MRI texture and shape in a machine learning scheme, Journal of Magnetic resonance in medicine, 62(6) (2009) 1609 -1618.

8. Kirchner M. and Fridrich J. J., On Detection of Median Filtering in Digital Images, Conference: Media Forensics and Security II, part of the IS\&T-SPIE Electronic Imaging Symposium, (N. D. Memon, Dittmann J., Alattar A. M., and Delp E. J., editors.), 7541 of Proceedings of SPIE, San Jose, CA, USA, 2010.

9. Oo S. Z. and Khaing A. S., Brain Tumor Detection and Segmentation Using Watershed Segmentation and Morphological Operation, International Journal of Research in Engineering and Technology, 3(3) (2014) 367-374.

10. Moldovanu S., Moraru L., Biswas A., Robust Skull-Stripping Segmentation Based on Irrational Mask for Magnetic Resonance Brain Images, J Digit Imaging, 28(6) (2015) 738747.

11. Ramesh M., Priya P., Arabi P.M., A Novel Approach for Efficient Skull Stripping Using Morphological Reconstruction and Thresholding Techniques, International Journal of Research in Engineering and Technology, 3(1) (2014) 96-101.

12. Senthilkumaran N. and Kirubakaran C., A Case Study on Mathematical Morphology Segmentation for MRI Brain Image, International Journal of Computer Science and Information Technologies, 5(4) (2014) 5336-5340.

13. Poleti E., Veronese E., Calabrese M., Bertoldo A., Grisan E., Supervised Classification of Brain Tissues through Local Multi-scale Texture Analysis by Coupling DIR and FLAIR MR Sequences, Proceedings SPIE Medical Imaging, San Diego, California, United States, 8314 pp. 83142T-83142T-7, 2012;

14. Moldovanu S., Moraru L., Bibicu D., Characterization of myocardium muscle biostructure using first order features, Digest Journal of Nanomaterials and Biostructures, 6(3) (2011) 1357-1365.

15. Johnson K. A. and Becker J. A., The Whole Brain Atlas, 1995, http://www.med.harvard.edu. 